\title{
Experimental Research on Signal Recognition Algorithm of Wireless Sensor Language
}

\author{
https://doi.org/10.3991/ijoe.v12i10.6203 \\ Harhenbek Muhaxov ${ }^{1,2}$, Zhong lou ${ }^{1}$, Wang Li $^{3}$, Tolewbek Samet ${ }^{4}$, Aheyeh Harhenbek ${ }^{5}$ \\ ${ }^{1}$ Wuhan University of technology, Wuhan, China \\ ${ }^{2}$ Xinjiang Applied Vocational Technical College, Kuitun, Xinjiang, China \\ ${ }^{3}$ Dalian Air Force Communications NCO School, Dalian, China \\ ${ }^{4}$ Altay Radio and Television University, Altay, Xinjiang, China \\ ${ }^{5}$ University of Science and Technology of China, Hefei, China
}

\begin{abstract}
In the past several decades, much research has been carried out on the wireless sensor network which is widely used in the fields of national defense and national economy within China. The main function of the language sensor is to transfer the voice signal into an electrical signal so as to facilitate the subsequent analysis and processing. Combined with the wireless network signal, it is widely used in banks, shopping malls, examination rooms, prisons, important places of military affairs and other places. This paper makes a detailed introduction of some theoretical knowledge of language recognition and puts forward the recognition algorithm in which the language signal is abstracted by language features. Afterwards, Matlab is used to make simulation in-depth research and classification based on Support Vector Machine. Finally, a large number of samples are collected for the experiment so as to research the effect of weighted feature value and structure of classification on speech recognition rate. The conclusion of the paper provides a basis for further subsequent theoretical study.
\end{abstract}

Index Terms-Wireless sensor, language recognition, voice features, support vector machine principle

\section{INTRODUCTION}

Wireless Sensor Network, WSN has the functions of signal collection, storage, processing, and transmission, etc. Many sensor nodes will construct a WSN which includes a variety of sensors including the ones for displaying information in the surrounding area, humidity, the size of the moving object, temperature, electromagnet, noise, earthquake, light intensity, pressure, soil composition, speed and direction. It can also be used in the battlefield, environment, disaster relief, industry and other fields[1].

Language is an important carrier of information. For understanding the real world and obtaining the features in the real world, the information can be acquired by acoustic signal as an alternative to visual observation. Therefore, as the most pervasive physical phenomenon in society, the application of language both in the battlefield and in real life has been highlighted[2]. currently. The acoustic target identification has been widely used in many fields. Currently, the recognition and application of acoustic target are based on the traditional pattern of a recognition algorithm[3]. The key to the technique is to extract some features of the signal of the acoustic target. Furthermore, the basic thought of acoustic target identification is processing the target and then identifying it so as to classify it into the possible category[4]. Research is being conducted on acoustic target in active battlefield environments.

Using wireless sensor networks to achieve language target recognition is a new technology with good application prospects. This paper uses the wireless sensor network platform to identify the language goal[5]. The target categories within the monitoring area can be displayed in real time on computers by building the language hardware platform, combining the recognition algorithm and the development and design of the upper computer software. The system can be used in an unattended military defensive environment. Moreover, this system also has wide application prospects in the civil application field, such as security monitoring, access control systems, etc[6].

\section{Structure Of Wireless Sensor Nodes AND PROCESS OF LANGUAGE RECOGNITION}

In different applications, the composition of sensor nodes is slightly different. But, the following several modules include the sensor module, wireless communication module, processing module and energy supply module, totalling four major parts, which is shown in Figure.1.

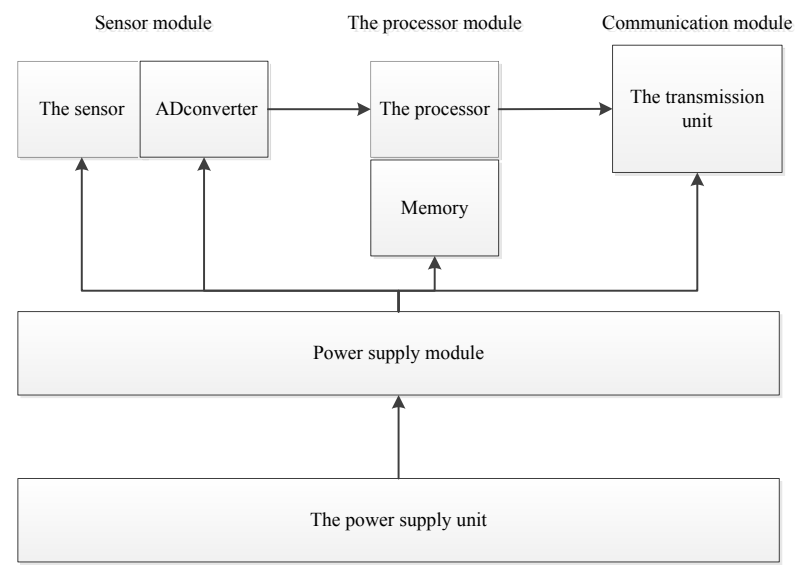

Figure 1. Node system diagram

This paper adopts a wireless sensor node based on JN5139 chip. It is a wireless microcontroller with low power consumption and low energy consumption. Its wireless protocol is widely used in ZigBee and its external can be used to stock application codes which will be implemented after entering internal RAM or during the process of operation. The equipment is integrated by hardware MAC, AES encryption acceleratorprocessor, ener- 


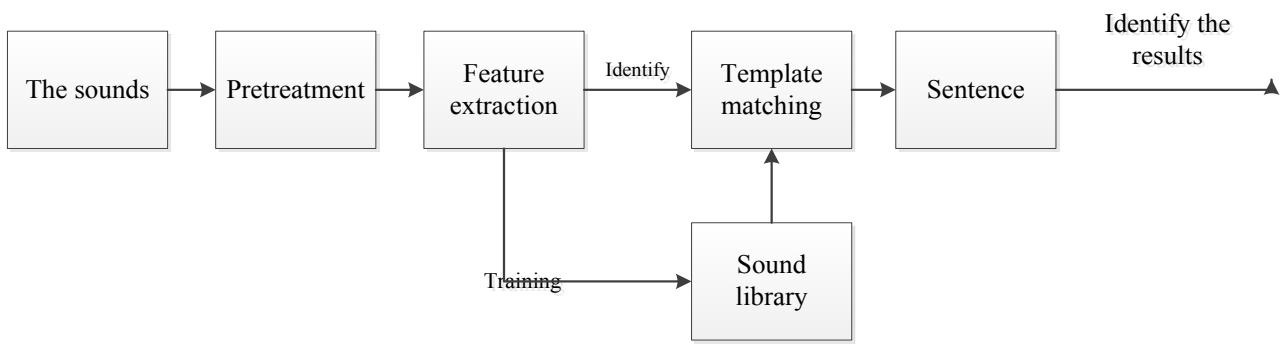

Figure 2. Voice recognition framework

gy-saving and timing for sleep mechanism, as well as a mechanism for guaranteeing safety and code encryption of the program. It has a wide application and it is suitable for safe and reliable wireless applications with low power, utility of the wireless sensor network based on IEEE802.15.4 and ZigBee systems[7].

The main process of acoustic target identification includes language acquisition, pre-processing, storage, feature extraction and recognition algorithm, as shown in Figure 2.

\section{LANGUAGE FEATURE EXTRACTION}

\section{A. Analysis of features}

In order to correctly and effectively carry out the classification and recognition of targets, the transfer of the acoustic signal is required so as to obtain the information which can reflect the categories of targets. The process of obtaining the signal features is also the process of feature extraction and feature selection[8]. Therefore, feature extraction is to transform the original language signal so as to obtain the original characteristics which can reflect the essence of the targets through a variety of transformations[9]. In terms of feature extraction for the signals of acoustic targets, the information which can be used to distinguish between a person, a vehicle, a gun and a tank is extracted.
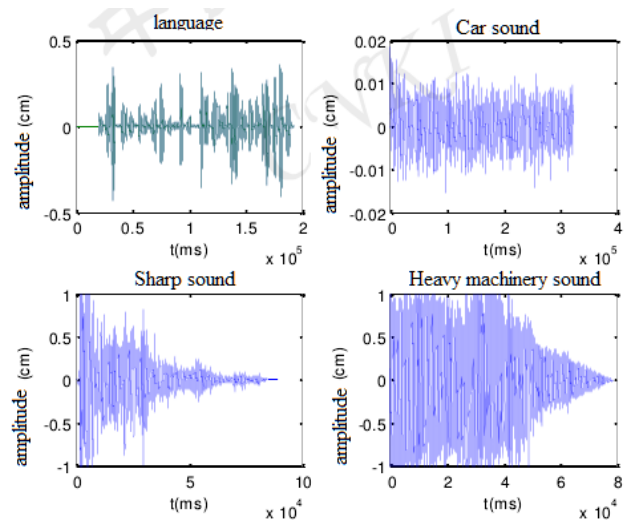

Figure 3. The waveform of language

Feature extraction methods include the features of energy, amplitude and zero-crossing rate based on time domain, feature of classical power spectrum based on frequency domain, features estimated by the power spectrum estimation of parameter model, the feature based on wavelet packet, etc. The feature of time domain is relatively simple and it can be calculated simply. As the resource of network node for wireless sensor is limited and not able to process very complex algorithm, this paper just compiles the feature of time domain in nodes, including three char- acteristics of short-time energy, short-time average amplitude and zero-crossing rate[10].

B. Analysis of time domain characteristics of language

1) Short term energy

$E_{t}=\sum_{n=0}^{N-1}\left|x_{t}(n)\right|^{2}$

In the equation, $\mathrm{N}--$ Frame length of language

Et--the value of energy in t time period

2) Short-time average amplitude

$E_{t}=\frac{1}{N} \sum_{n=0}^{N-1}\left|x_{t}(n)\right|$

In the equation, N--Frame length of language

Et--the value of average amplitude in t time period

3) Zero-crossing rate

$Z c r=\frac{1}{2 N} \sum_{n=0}^{N-1}|\operatorname{sgn}[x(n)]-\operatorname{sgn}[x(n-1)]|$

In the equation: $\operatorname{sgn}[x(n)]=\left\{\begin{array}{l}+1^{x(n) \geq 0} \\ -1_{x(n) \leq 0}\end{array}\right.$

C. Language simulation diagram
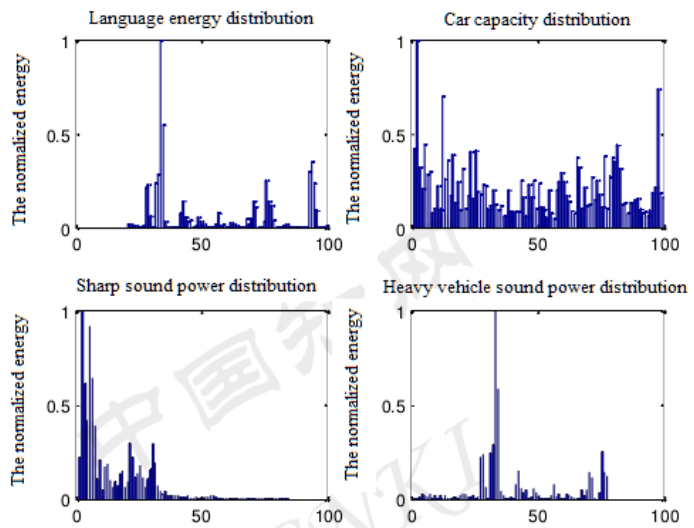

Figure 4. Energy distribution of language

\section{1) Time domain waveform of language}

In order to observe the time domain of language, this paper puts forward the time domain waveform of language, as shown in Figure 3.

\section{2) Energy distribution of language}

In order to observe the energy distribution of language, this paper puts forward the diagram of energy distribution of language [11]. It is able to more intuitively show the 


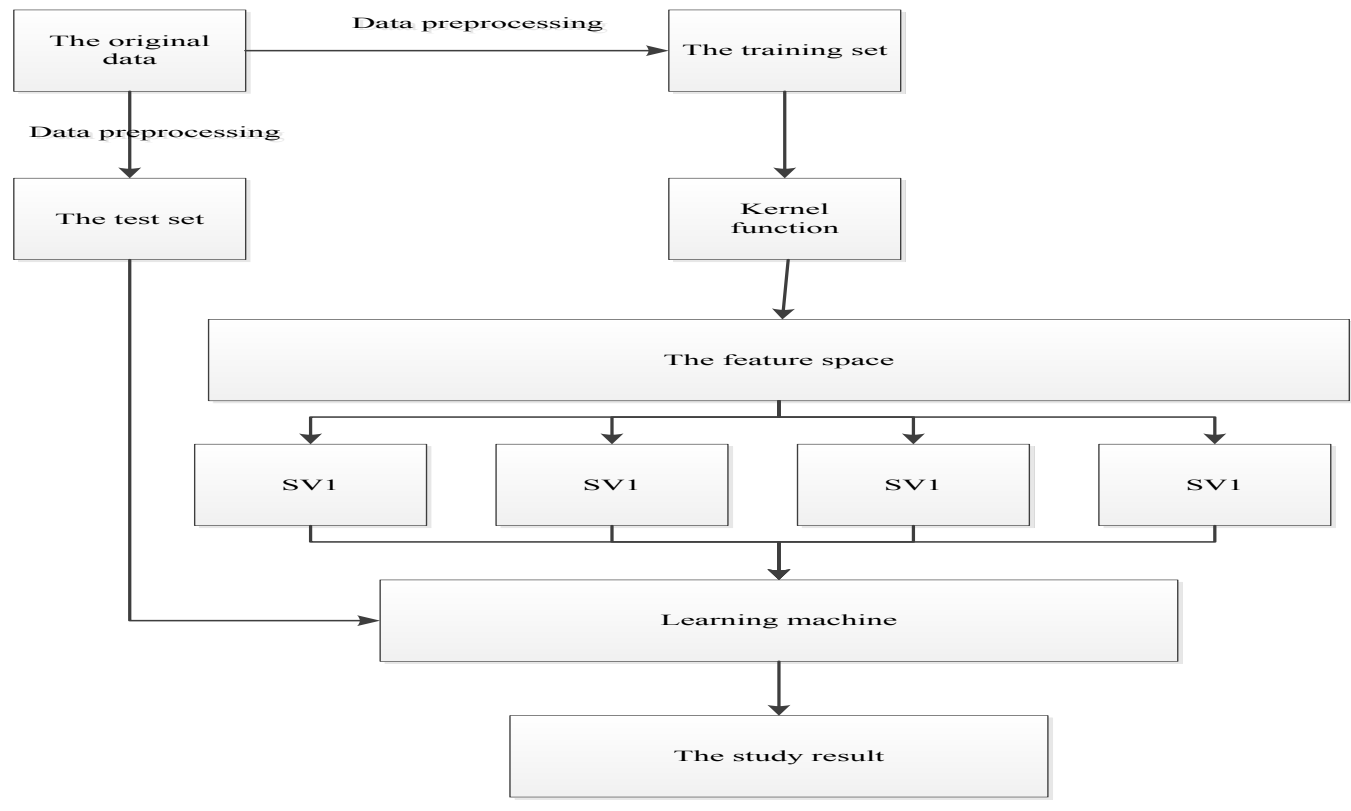

Figure 5. Working sketch

energy distribution of each language target with its energy being normalized within $[0,1]$ so that it can be observed that the corresponding energy of different frames with different targets are different, which is conductive for identifying and processing later. This is shown in Figure 4.

\section{SupPort Vector MAchine Algorithm}

The support vector machine algorithm is a method for the study of machines, and it is endowed with inherent risk of experience and minimum scope of confidence. The basic principle of the support vector machine is to determine an optimal hyper plane which will make two kinds of targets have the maximum classification interval[12]. Optimal classification hyper plane with the maximum structural interval is regarded as the decision function. Its structure of working principle for classification is shown Figure 5.

In terms of its calculation principle, there are two types of samples which can be divided[13].

$$
\left(x_{1}, y_{1}\right),\left(x_{2}, y_{2}\right) \ldots \ldots\left(x_{n}, y_{n}\right), x \in R^{n}, y \in\{-1,1\} \text { are the }
$$
category markers of the targets. In order to determine the plane which will distinguish the two categories, the hyper plane is recorded as $\left(\omega^{*} \mathrm{x}\right)+\mathrm{b}=0$. Under the rule of minimization of empirical risk, there are numerous such kinds of hyper planes, but in terms of the training sample, some hyper planes have better effect of classification than others. The core idea of support vector machine is that the larger the classification interval is, the better the promotional ability will be[14-15].

In order to distinguish the target effectively, it must meet:

$$
\begin{aligned}
& \left(\omega *^{X_{i}}\right)+b \geq 1, y_{i}=1 \\
& \left(\omega *^{X_{i}}\right)+b \geq 1, y_{i}=-1
\end{aligned}
$$

The above two equations can be integrated into a normalizing condition $y_{i}\left[\left(\omega *^{X_{i}}\right)+b\right] \geq 1, i=1,2, \ldots n$ and the distance of each sample to hyper plane is:

$$
k=\frac{|\omega * x+b|}{\|\omega\|}
$$

So, the classification interval $=\min _{+1} \frac{|\omega * x+b|}{+2 \min _{-1}} \frac{|\omega * x+b|}{\|\omega\|}$
$=\frac{1}{\|w\|}\left(\min _{y=1}|w * x+b|+\min _{y=-1}|w * x+\not b y|=\frac{2}{\|\omega\|}\right.$ In this way, $\frac{1}{2}\|\omega\|^{2}$ will be the minimum.

In the optimum classification plane, in order to make all the samples classify correctly with the maximum classification interval, the conditions in equation (6) should be met. Therefore, in terms of determining the optimum solution, it can be regarded as a simple optimization problem.

$$
\min \frac{1}{2}\|\omega\|^{2}=\frac{1}{2}(\omega * \omega)
$$

That is to say:

$$
\min \frac{1}{2}\|\omega\|^{2}=\frac{1}{2} \min
$$

At the same time, the constraint condition in the above normalization (7) should be met; that is $y i[\omega * x+b] \geq 1, i=1,2, \ldots n$, when $\mathrm{y}=1$, it is classified as one category, when $y=-1$, it is categorized as another category.

$\alpha\left(\alpha_{1} \alpha_{2} \ldots \alpha_{n}\right)\left(\alpha_{n} \geq 0\right)$ is introduced; that is to say, the problem of quadratic programming problem of minimization is actually the problem of minimization in the original constraint condition.

$$
\min [L(w, b, a)]=\frac{1}{2}\|w\|^{2}-\sum_{i=1}^{n} \alpha_{i}\left\{y_{i}\left[\omega * x_{i}+b\right]-1\right\}
$$

Its partial derivation is calculated and its value set as 0 ; that is:

$$
\frac{\partial L(\omega, b, a)}{\partial \omega}=\omega-\sum_{i=1}^{n} y_{i} \alpha_{i} x_{i}=0
$$




$$
\frac{\partial L(\omega, b, a)}{\partial \omega}=\sum_{i=1}^{n} y_{i} \alpha_{i}=0
$$

After calculating the above equation, the results will be substituted into the original lagrange function and the result is:

$$
L(\alpha)=\sum_{i=1}^{n} \alpha_{i}-\frac{1}{2} \sum_{i=1}^{n} \alpha_{i} \alpha_{j} y_{i} y_{j}\left(x_{i} * x_{j}\right)
$$

If the function $\mathrm{W}(\alpha)$ is the maximum

$$
\max W(\alpha)=\max \{\min L(\omega, b, \alpha)\}=\max \left\{\sum_{i=1}^{n} \alpha i-\frac{1}{2} \sum_{i=1}^{n} \alpha_{i} \alpha_{j} y_{i} y_{j}\left(x_{i} * x_{j}\right)\right\}
$$

The equation: $\sum_{i=1}^{n} \alpha_{i} y_{i}=0, \alpha_{i} \geq 0, i=1,2, \ldots n$

According to the above equation $w=\sum \alpha_{i} y_{i} x_{i}$ can be obtained and the final optimal classification function obtained is:

$$
f(x)=\operatorname{sgn}\{(w * x)+b\}=\operatorname{sgn}\left(\sum y_{i} \alpha_{i}\left(x_{i}, x\right)+b\right)
$$

In the equation, $\mathrm{b}$ is the bias, according to $3-15$, thus yielding:

$$
b=\frac{1}{2}[w * x(1)+(w * x(-1)]
$$

\section{Structure And Computational Principle OF MULTI-ClASS CLASSIFIER}

The support vector machine is dominated by two categories of classification. If the multi-class classification is to be carried out, the multi-class classifier must be established[16]. This paper carries out research on four multiclass classifiers of one-on-one, one-on-many, two fork tree-SVM multiple classification method and decisiondirected acyclic graph respectively. In the following content, the algorithms of these classifiers are introduced.

\section{1) One-on-one method}

The basic principle of one-on-one structure is that in $n$ categories of samples, a classifier is created for every two samples. Afterwards, these classifiers are trained respectively with the testing sample being input after the training[17]. Every sample will be tested in these classifiers and each classifier will obtain an identification result. All of the identification results will be voted on and the samples with the most votes are the category they belong to.

\section{2) One-on-many method}

The principle of one-on-many algorithm is that in $\mathrm{n}$ categories of samples, $n$ classifiers are created. When creating the classifier of the first class of samples, its training samples are regarded as one category which is marked as 1 while the other training samples are regarded as another category which will be marked as -1 . When testing the samples, the voting method is adopted. The testing sample will be input into $n$ classifiers and then $n$ classification results will be obtained. The results will be voted on afterwards, and the samples with the most votes are the category they belong to.

3) Two fork tree-SVM multiple classification method

The principle of two fork tree-SVM multiple classification method is that there are a training period and a testing period[18]. When training, it starts from the leaf node and then moves towards the root node. Each time, a category will be formed until it reaches the root node. The testing starts from the root node, which is carried out by adopting preorder traversal two fork tree until it reaches the leaf node. The category of the leaf node is in the category of the testing sample. Figure 6 shows the structural diagram of the two fork tree.

\section{4) Decision-directed acyclic graphs}

The decision-directed acyclic graph must create n(n1) $/ 2$ classifiers. For a classification problem of $n$ category, there are $n(n-1) / 2$ nodes[19]. There are two categories for each classifier which is distributed in the structure of $n$ layers. On the top layer, only one node is included, also referred to as the root node, while in the second layer there are two nodes. In ith layer, there are $i$ nodes. In these nodes, the ith node in jth layer pints to the ith node and $(i+1)$ th node in $j+1$ layer. In the training process of the DDAG algorithm, only the sub-classifier is trained. The error rate can be reduced by maximizing the interval of the two-valued classifier. The structure of the DDAG classifier is different from the three classifier. In the general decision of the three methods, when some node is mistaken, the error will last until the end of the training. As there is redundancy in the DDAG structure, the ratio of classification path of the same kind must be the same. For the three common decisions, its calculation is simple and highly efficient. Figure 7 shows the structural diagram of the decision-directed acyclic graph.

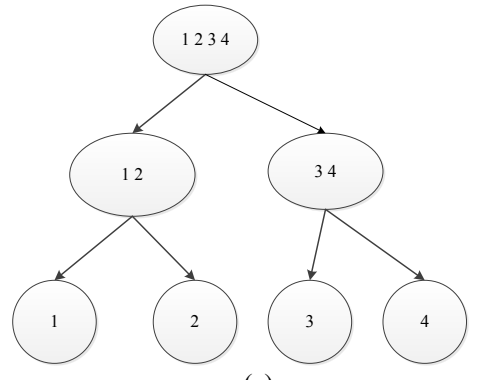

(a)

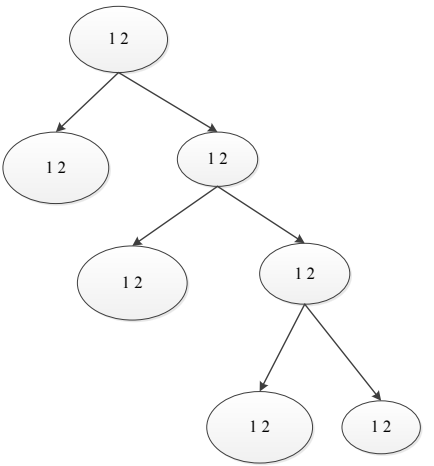

(b)

Figure 6. Binary tree structure

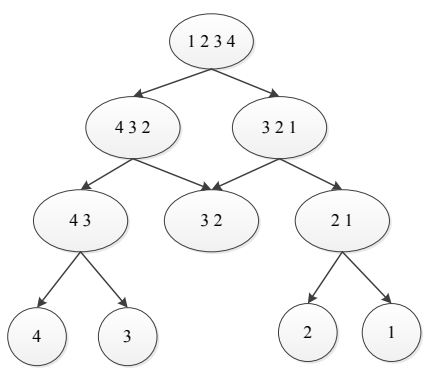

Figure 7. Decision-directed acyclic graph 
PAPER

EXPERIMENTAL RESEARCH ON SignAl RECOGNITION ALgORIthM OF WiRELESS SENSOR LANGUAGE

TABLE I.

INFLUENCE OF THE AVERAGE AMPLITUDE OF RECOGNITION RATE

\begin{tabular}{|c|c|c|c|c|c|}
\hline Average amplitude & Language & Car sound & Sharp sound & Heavy duty vehicle & On average \\
\hline 800 & $55 \%$ & $77 \%$ & $45 \%$ & $81 \%$ & $65.2 \%$ \\
\hline 8000 & $55 \%$ & $87 \%$ & $51 \%$ & $84 \%$ & $69.4 \%$ \\
\hline 80000 & $68 \%$ & $89 \%$ & $57 \%$ & $95 \%$ & $77.4 \%$ \\
\hline 800000 & $71 \%$ & $92 \%$ & $72 \%$ & $95 \%$ & $82.6 \%$ \\
\hline 2000000 & $78 \%$ & $94 \%$ & $80 \%$ & $95 \%$ & $85.7 \%$ \\
\hline 60000000 & $75 \%$ & $96 \%$ & $70 \%$ & $95 \%$ & $84.2 \%$ \\
\hline
\end{tabular}

TABLE II.

INFLUENCE OF A ZERO RATE OF RECOGNITION RATE

\begin{tabular}{|c|c|c|c|c|c|}
\hline Zero-crossing rate & Language & Car sound & Sharp sound & Heavy duty vehicle & On average \\
\hline 300 & $55 \%$ & $77 \%$ & $45 \%$ & $82 \%$ & $65.2 \%$ \\
\hline 3000 & $59 \%$ & $81 \%$ & $54 \%$ & $92 \%$ & $71.4 \%$ \\
\hline 30000 & $73 \%$ & $82 \%$ & $78 \%$ & $95 \%$ & $79.4 \%$ \\
\hline 300000 & $86 \%$ & $97 \%$ & $83 \%$ & $96 \%$ & $82.6 \%$ \\
\hline 3000000 & $89 \%$ & $84 \%$ & $89 \%$ & $97 \%$ & $85.7 \%$ \\
\hline 30000000 & $84 \%$ & $80 \%$ & $93 \%$ & $97 \%$ & $84.2 \%$ \\
\hline
\end{tabular}

TABLE III.

INFLUENCE OF WEIGHTED SHORT-TIME ENERGY FOR THE RECOGNITION RATE

\begin{tabular}{|c|c|c|c|c|c|}
\hline Weighted energy & Language & Car sound & Sharp sound & Heavy duty vehicle & On average \\
\hline 10 & $55 \%$ & $77 \%$ & $49 \%$ & $80 \%$ & $65.2 \%$ \\
\hline 20 & $55 \%$ & $77 \%$ & $49 \%$ & $80 \%$ & $65.2 \%$ \\
\hline 30 & $55 \%$ & $77 \%$ & $49 \%$ & $80 \%$ & $65.2 \%$ \\
\hline 100 & $55 \%$ & $77 \%$ & $49 \%$ & $80 \%$ & $65.2 \%$ \\
\hline 1000 & $55 \%$ & $77 \%$ & $49 \%$ & $80 \%$ & $65.2 \%$ \\
\hline 100000 & $55 \%$ & $77 \%$ & $49 \%$ & $80 \%$ & $65.2 \%$ \\
\hline
\end{tabular}

TABLE IV.

INFLUENCE OF WEIGHTED SHORT-TIME ENERGY FOR THE RECOGNITION RATE

\begin{tabular}{|c|c|c|c|c|c|}
\hline Amplitude and Zero-crossing rate & Language & Car sound & Sharp sound & Heavy duty vehicle & On average \\
\hline 60000004000000 & $89 \%$ & $80 \%$ & $81 \%$ & $96 \%$ \\
\hline 60000002000000 & $86 \%$ & $85 \%$ & $80 \%$ & $97 \%$ \\
\hline 50000002000000 & $91 \%$ & $79 \%$ & $80 \%$ & $95 \%$ \\
\hline 50000003000000 & $85 \%$ & $77 \%$ & $79 \%$ & $92 \%$ \\
\hline $900000 \quad 3000000$ & $89 \%$ & $81 \%$ & $83 \%$ & $94 \%$ \\
\hline 400000 & 2000000 & $83 \%$ & $85 \%$ & $79.2 \%$ \\
\hline
\end{tabular}

TABLE V.

INFLUENCE OF WEIGHTING OF AVERAGE SHORT-TERM AMPLITUDE, ZERO-CROSSING RATE AND ENERGY WEIGHT AT THE SAME TIME

\begin{tabular}{|c|c|c|c|c|c|}
\hline $\begin{array}{c}\text { Amplitude, Zero-crossing, energy } \\
\text { weight }\end{array}$ & Language & Car sound & Sharp sound & $\begin{array}{l}\text { Heavy duty } \\
\text { vehicle }\end{array}$ & On average \\
\hline $50000003000000 \quad 10$ & $90 \%$ & $82 \%$ & $81 \%$ & $97 \%$ & $87.2 \%$ \\
\hline 6000000200000020 & $88 \%$ & $86 \%$ & $80 \%$ & $97 \%$ & $89.2 \%$ \\
\hline $50000002000000 \quad 10$ & $93 \%$ & $82 \%$ & $80 \%$ & $97 \%$ & $87.2 \%$ \\
\hline $50000003000000 \quad 10$ & $86 \%$ & $79 \%$ & $80 \%$ & $98 \%$ & $88.2 \%$ \\
\hline $900000 \quad 300000020$ & $92 \%$ & $82 \%$ & $82 \%$ & $97 \%$ & $83.2 \%$ \\
\hline $400000 \quad 2000000 \quad 15$ & $88 \%$ & $86 \%$ & $81 \%$ & $95 \%$ & $86.2 \%$ \\
\hline
\end{tabular}




\section{EXPERIMENTAL RESEARCH ON SENSOR NODE}

\section{A. Experimental equipment, environment and content}

1 The experimental equipment includes:

(1). A sensor node for collection of language and a coordinator node;

(2). A language box used to simulate the language of guns and tanks;

(3). A laptop;

2 The experimental environment of this paper is on a road outdoors with low noise. This paper does not consider background noise.

\section{Experimental content}

The experiment is carried out with a single node. On the side of the road, a node for collecting language and a coordinator node are placed. The language of a person and vehicles are collected in real life while the language of tanks and guns are simulated by the language box. It collects the language characteristics of the four targets which are person, vehicle, guns and tanks whose language will be transmitted to the sensor node. The sensor node will extract and send the characteristic quantity of language to the coordinator after processing it. The coordinator will then send it to a serial assistant passing through the serial port. The four targets will collect 200 samples respectively including 100 training samples and 100 identification samples, after which the following research on parameters is carried out.

\section{B. The effect of weighted results of different eigenvalues} on recognition rate of speech

\section{1) Weighting of short-time average amplitude}

Kernel function selects Ker="rbf" with the parameter of $\mathrm{P} 1=2$ and $\mathrm{C}=1000$. The classifier of the support vector machine is the structure of a two fork tree. When other parameters remain unchanged and only the weight of the short-time average amplitude is changed, the change for recognition rate is shown in Table 1.

The results of the above experiment show that within a certain scope, when the weight of the short-time average amplitude is increased, the recognition rate is also increased. Furthermore, when it reaches a certain parameter, it decreases accordingly. When the short-time average amplitude is at about 800,000 , the average recognition rate of the four targets reaches the maximum value.

\section{2) Weight of zero-crossing rate}

The kernel function selects Ker="rbf" and the parameters are $\mathrm{P} 1=2$ and $\mathrm{C}=1000$. The classifier of the support vector machine is the structure of two fork tree. When other parameters remain unchanged and only the weight of zero-crossing rate is changed, the change of recognition rate is shown in table 2 .

The experiment shows that within a certain scope, when the weight of zero-crossing rate is increased, the recognition rate is also increased. Furthermore, when it reaches a certain value, it decreases accordingly. Although the situation varies, the overall trend is the same. When zerocrossing rate is at about $3,000,000$, the average recognition rate of the four targets reaches the maximum value.

3) Weighting of short-time energy

The kernel function selects Ker="rbf" and the parameters are $\mathrm{P} 1=2$ and $\mathrm{C}=1000$. The classifier of the support vector machine is the structure of a two fork tree. When other parameters remain unchanged and only the weight of energy is changed, the situation of change for recognition rate is shown in Table 3.

The above experiment shows that if only the weight of energy is changed, the recognition rate remains the unchanged.

4) The weighting of short-time average amplitude and zero-crossing rate are at the same time.

The kernel function selects Ker="rbf" and the parameter $\mathrm{P} 1=2$ and $\mathrm{C}=1000$. The classifier of the support vector machine is the structure of a two fork tree. When other parameters remain unchanged and only the weight of energy is changed, the change of recognition rate is shown in Table 4.

The experiment shows that when the weight of amplitude is $5,000,000$ and the weight of zero-crossing rate is $2,000,000$, the average recognition rate of the four targets reaches the maximum of $89 \%$.

5) In weighting of average short-term amplitude, the zero-crossing rate and energy weight are considered at the same time.

The kernel function selects Ker="rbf" and the parameters are $\mathrm{P} 1=2$ and $\mathrm{C}=1000$. The classifier of the support vector machine is the structure of the two fork tree. When other parameters remain unchanged and only the weight of energy is changed, the change for recognition rate is shown in Table 5 .

The above experiment shows that although there is no effect in the improvement of recognition rate if just the energy is weighted, the situation in which three characteristics are weighted at the same time has a better effect than weighing for just one or two characteristics. The overall effect can be heightened by $0.25 \%$.

\section{CONCLUSION}

A wireless sensor network is widely used in various fields. This paper builds a platform of a recognition system for an acoustic target based on a wireless sensor network by combining the wireless sensor network with language recognition. It conducts research on the language recognition of a person, a vehicle, guns and tanks. These four categories are targets, and the extraction methods and realization in sensor nodes, as well as recognition of the algorithm of support vector machine and a variety of structures of multi-class classifier. Afterwards, the joint identification algorithm for multiple sensor nodes is studied and then the experimental test of a single node is carried out resulting in weighting of different characteristic values on identification rate being analyzed. The following conclusions are obtained.

1. A platform of recognition system for acoustic target based on wireless sensor network is established which includes compiling the programs of language collection, characteristics extraction and the interface design of the upper computer. Finally, the category of the target region can be displayed in real time for observation.

2 Research on joint decision algorithm of multiple nodes includes the analysis of the advantages and disadvantages of voting judgment and energy judgment... Furthermore, based on the two algorithms, the improved voting judgment and energy judgment are put forward which can be used more widely theoretically. 
3 Research on identification of sensor node includes the recognition algorithm of support vector machine being studied with the four structures of multi-class classifiers which include the structure of one-on-one, one-on-many, and the structure of two fork tree multi-class classifications and the structure of decision-directed acyclic graph being researched. Further experiments were also conducted. After the experiment and research on the effect of each parameter on identification rate, it shows that the average identification rate of the structure of decision-directed acyclic graph is better comparative to its identification rate being up to $93.5 \%$.

\section{REFERENCES}

[1] H. Wu, M.M. Jerry, "Multicategory classification of ground vehicles based on their Acoustic emissions", Proc. of SPIE, pp.3142, 2004. http://dx.doi.org/10.1117/12.541700

[2] R.R. Brooks, P. Ramathan, and A.M. Sayeed, "Distributed Target Classification and Tracking in Sensor Networks", Proceeding of the IEEE, pp.116-137, 2003. http://dx.doi.org/10.1109/jproc. 2003.814923

[3] P.M. Georgios, N.A. John, "Vehicle Classification in Sensor Networks using time-domain Signal processing and Neural Networks", Microprocessors and Micro-systems, pp.31-42,2007.

[4] R. Cabell, "The identification of helicopter AIAA 13th Aero acoustics Conference", 199 C noise using anural network, pp.30ロ48, 2010.

[5] M. Georgios, A. John, "Lightweight Time Encoded Signal Processing for Vehicle Recognition in Sensor Networks", Research in Microelectronics and Electronics, pp.47-50, 2006.

[6] E.W. Peter, W.H. Michael, "Efficient sensor network vehicle classification using peak Harmonies of acoustic emissions", Pro. of SPIE, pp.1-12, 2008.

[7] K. Sohrabi, "Protocols for self-organization of a wireless sensor network", IEEE Personal Communications, Vol.(5),pp.16-27, 2010.

[8] C. Weihsu, C. Jenlin, "A Practical Guide to Support Vector Classification", IEEE Personal Communications, Vol.(5), pp.19-27, 2010.

[9] B. Sun, S.J. Song and C. Wu, "A new algorithm of support machine based on weighted feature". International Conference on Machine Learning and Cybernetics, Vol.3, pp.16-27, 2009.

[10] H.J. Xing, D.Z. Tian, "A novel support vector machine with its features weighted by mutual information", Proceeding of the 2008 International Joint Conference on Neural Networks, Vol.6, pp.1631. 2008.

[11] D. Yeung, D. Wang, "Structured large margin machine: sensitive to data distribution", Machine Learning, Vol.68, pp. 171-200. 2007. http://dx.doi.org/10.1007/s10994-007-5015-9

[12] M. Georgios, A. John, "Light weight Time Encoded Signal Processing for Vehicle Recognition in Sensor Networks", Research in Microelectronics and Electronics, pp.47-50, 2006.

[13] M.F. Duarte, Y.H.Hu, "Vehicle classification in distributed sensor networks", Journal of Par8llel and Distributed Computing, pp.2638, 2004.
[14] H.D. Ma, D. Tao, "Multimedia sensor network and its research progresses", Journal of Software, Vol.2009, pp.13-28, 2012.

[15] Z. Rui, W. Wenjian, "Facilitating the applications of support vector machine by using a new kernel", Expert Systems with Applications, pp.14-30, 2011.

[16] B. Sun, S.J. Song and C. Wu, "A new algorithm of support machine based on weighted feature", International Conference on Machine Learning and Cybernetics, Vol.3, pp.16-27, 2009.

[17] H.J. Xing, D.Z. Tian, "A novel support vector machine with its features weighted by mutual information", Proceeding of the 2008 international Joint Conference on Neural Networks, pp.16-31, 2008.

[18] D.S. Yeung, D.F. Wang, and X.Z. Wang, "Structured large margin machine: sensitive to data distribution", Machine Learning, Vol.68, pp.171-200, 2007. http://dx.doi.org/10.1007/s10994-0075015-9

[19] H.D. Ma, D. Tao, "Multimedia sensor network and its research progresses", Journal of Software, Vol.9, pp.13-28, 2009.

\section{AUTHORS}

Harhenbek Muhaxov is an associate professor in Xinjiang Applied Vocational Technical College, Arts department, Xinjiang Applied Vocational Technical College, Kuitun, Xinjiang, 833200 China. His research directions are turbo coding and decoding, neural networks and their applications and wireless communications. He is also with School of Computer Science and Technology, Wuhan University of technology, Wuhan, 430070 China (e-mail: harhenbek@163.com).

Zhong Lou, is a professor at the School of Computer science and Technology, Wuhan University of Technology, Wuhan, 430070 China. His research directions are intelligent method and intelligent systems, knowledge discovery and data mining, software engineering techniques and approaches. (email: zhongluo@whut.edu.cn)

Wang Li is a lecturer in department of Radio Navigation, Dalian Air Force Communications NCO School, Dalian, 116600 China. Her research directions are radio navigation, system integration and intelligent method. (email: wangliwlair@126.com)

Tolewbek Samet is an associate professor in Altay Radio and Television University, Altay, Xinjiang, 836500 China. His research directions are computer application technology. (email: gulsara@163.com)

Aheyeh Harhenbek is an undergraduate in school of Computer science and technology, University of Science and Technology of China, Hefei, 230027 China. His research direction is Speech information processing. (email: AhyehHarhenbek@163.com).

Submitted 03 September 2016. Published as resubmitted by the authors 15 October 2016. 